\title{
A beam expander facility for studying $x$-ray optics
}

Christensen, Finn Erland; Hornstrup, Allan; Frederiksen, P.; Nilsson, C.; Grundsøe, Peter; Ørup, P.; Jacobsen, E.; Schnopper, H. W.; Lewis, R.; Hall, C.

Published in:

Review of Scientific Instruments

Link to article, DOI:

$10.1063 / 1.1143124$

Publication date:

1992

Document Version

Publisher's PDF, also known as Version of record

Link back to DTU Orbit

Citation (APA):

Christensen, F. E., Hornstrup, A., Frederiksen, P., Nilsson, C., Grundsøe, P., Ørup, P., Jacobsen, E.,

Schnopper, H. W., Lewis, R., \& Hall, C. (1992). A beam expander facility for studying x-ray optics. Review of Scientific Instruments, 63(1), 1168-1171. https://doi.org/10.1063/1.1143124

\section{General rights}

Copyright and moral rights for the publications made accessible in the public portal are retained by the authors and/or other copyright owners and it is a condition of accessing publications that users recognise and abide by the legal requirements associated with these rights.

- Users may download and print one copy of any publication from the public portal for the purpose of private study or research.

- You may not further distribute the material or use it for any profit-making activity or commercial gain

- You may freely distribute the URL identifying the publication in the public portal

If you believe that this document breaches copyright please contact us providing details, and we will remove access to the work immediately and investigate your claim 


\title{
A beam expander facility for studying $x$-ray optics
}

\author{
F. E. Christensen, A. Hornstrup, P. Frederiksen, C. Nilsson, \\ P. Grundsøe, P. Qrup, E. Jacobsen, and H. W. Schnopper \\ Danish Space Research Institute, Gl. Lundtoftevej 7, DK-2800 Lyngby, Denmark
}

R. Lewis and C. Hall

Daresbury Laboratory, Daresbury, Warrington WA4 4AD, United Kingdom

(Presented on 17 July 1991)

\begin{abstract}
The detailed study of the performance of full scale $x$-ray optics often requires the illumination of large areas. This paper describes a beam expander facility at the Daresbury Synchrotron Radiation Facility. It combines monochromatization and beam expansion in one dimension. The beam expansion is obtained from an extremely asymmetric reflection in a large single crystal of $\mathrm{Si}$. An expansion of a factor of 50 was obtained in one dimension. The expanded beam of $\sim 85 \mathrm{~mm}$ is limited only by the crystal size. The facility is installed in a 12-m-long hutch. A specific application, in which a high throughput $\mathrm{x}$-ray telescope will be studied, is described in detail.
\end{abstract}

\section{INTRODUCTION}

It is of crucial importance for the development of advanced $\mathrm{x}$-ray optics and detectors, that testing and experimentation can be performed under realistic conditions over a wide range of $x$-ray energies. For large $x$-ray devices such as two-dimensional position sensitive detectors (PSD), ${ }^{1}$ grazing incidence high throughput $\mathrm{x}$-ray telescopes (XSPECT/SODART, ${ }^{2}$ ASTRO-D, ${ }^{3}$ JET-X, ${ }^{4}$ and $\mathrm{XMM}^{5}$ telescopes) and crystal spectrometers [the $\sim 1000$ $\times 600 \mathrm{~mm}^{2}$ objective crystal spectrometer ${ }^{6}$ (OXS), to be flown on the SPECTRUM-X-GAMMA (SRG) satellite] the illumination of large areas with a suitably monochromatized and collimated beam is required. A pencil beam which is both monochromatized and collimated, is readily available over a wide energy range from synchrotrons and conventional $x$-ray sources. The testing of large area devices with such a beam is, however, very time consuming and requires an elaborate, two-dimensional, scanning apparatus.

The broad continuum of synchrotron radiation is extremely useful. For example, the efficiency of most x-ray devices is a function of wavelength and there are discontinuities which occur at absorption edges or emission features of the materials in reflectors, transmission windows, and detectors. In many cases, at least one or more features occur within the useful operating energy range. It is very difficult to accurately calibrate the device near such a feature with the limited number of emission line energies, which are available from either radioactive or $\mathrm{x}$-ray tubes. Synchrotron light is linearly polarized in the plane of the electron orbit and the beam expander can be used to evaluate the polarization sensitivity of crystal spectrometers, ${ }^{6}$ polarimeters, ${ }^{7}$ and x-ray optics over a wide range of energy.

The working energy range covered by the telescopes which will be flown in the period from 1994 to 2000 (ASTRO-D, SRG, JET-X \& XMM) is from $\sim 0.1$ to $\sim 25$ $\mathrm{keV}^{2-5}$ All of them are based on the Wolter-1 design ${ }^{8}$ which allows accurate images to be recorded with a high signal to noise ratio by focusing the $\mathrm{x}$ rays on relatively small pixels in a position sensitive $x$-ray detector. These $\mathrm{x}$-ray telescopes are designed to yield images with an angular resolution of a few arcmin or less. The design resolution of ASTRO-D ${ }^{3}$ and SRG $^{2}$ is $\sim 120$ arcsec, of JET-X ${ }^{4}$ $\sim 20$ arcsec, and of $X_{M M} M^{5} \sim 30$ arcsec. $A$ beam expander with good collimation and which can provide a beam wide enough to cover the radial aperture of these telescopes in the energy range from 5 to $15 \mathrm{keV}$ will, therefore, be extremely useful.

\section{THE BEAM EXPANDER}

The principle of the device is based upon the one-dimensional expansion of a beam which undergoes extremely asymmetric reflection in a perfect single crystal of Sit. The most efficient use of the crystal in terms of the width of the expanded beam versus the length of the crystal required, is obtained with a Bragg angle in the vicinity of $45^{\circ}$. The beam from the synchrotron is polarized in the horizontal plane and, as a compromise, a Bragg angle of $\sim 60^{\circ}$ has been chosen for the first experiments. Since the horizontal source dimension is $2.8 \mathrm{~mm}$ [full width at half-maximum (FWHM)] at the Daresbury Synchrotron, ${ }^{9}$ it is necessary to expand the beam by a factor of 50-100 to get an expanded beam of $100-200 \mathrm{~mm}$ which is our design goal. A premonochromator avoids higher order contamination. It is shown together with the beam expander in Fig. 1.

The testing of optics with focal lengths in the range 3-8 $\mathrm{m}$, such as the high throughput $\mathrm{x}$-ray telescopes mentioned above, imposes the need for an adequately large work area. The premonochromator is located in a prehutch. It is a standard, double-reflection, $\mathrm{Si}(111)$ channelcut crystal and is oriented to reflect in the vertical plane. The main hutch (hutch 8.4) contains the beam expander and the optics to be tested. The monochromator and the beam expander crystal define a $(n,-n)$ double reflection system where the same reflection is used in both crystals. The beam from the monochromator strikes the beam expander crystal at a small grazing angle $\left(\sim 1^{\circ}\right)$ relative to the surface. The reflecting planes make an angle $\phi$ relative to the surface and a beam of width $H$ makes a footprint on 


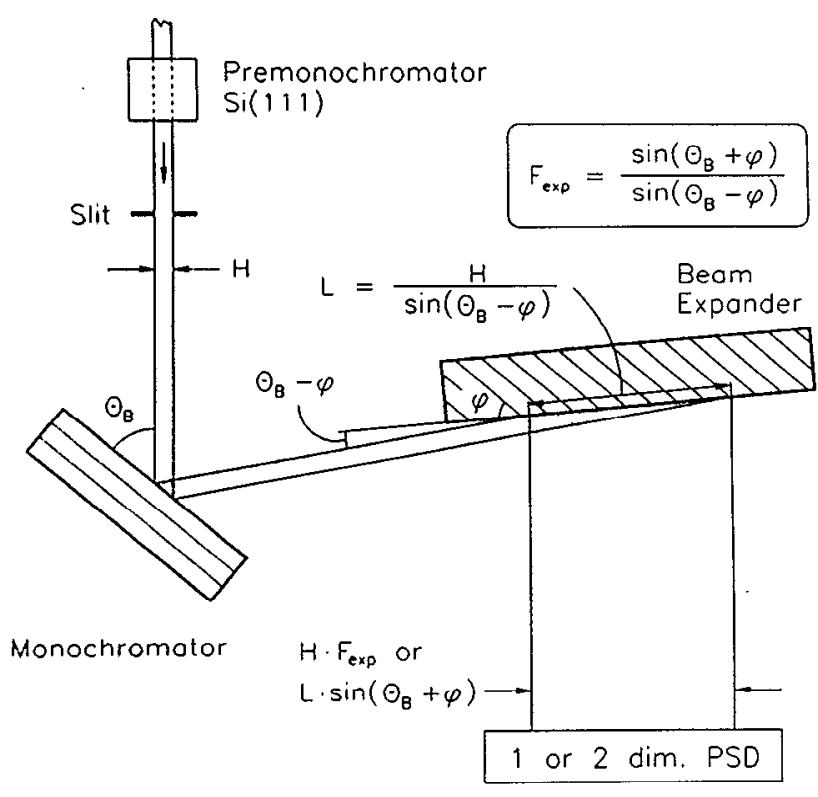

FIG. 1. A topview of the beam expander unit. A premonochromatized beam of horizontal width $H$ is reflected in the symmetric monochromator crystal followed by beam expansion in the extremely asymmetricly cut beam expander crystal.

the beam expander crystal of $L \equiv H / \sin \left(\theta_{B}-\phi\right)$ where $\theta_{B}$ is the Bragg angle of the reflection. The width of the expanded beam is $H \sin \left(\theta_{B}+\phi\right) / \sin \left(\theta_{B}-\theta\right)$ or $L \sin \left(\theta_{B}+\theta\right)$. The factor $\sin \left(\theta_{B}+\phi\right) / \sin \left(\theta_{B}-\phi\right)$ defines the beam expansion factor $F_{\text {exp }}$ and plays a central role in the classical theory of dynamical x-ray diffraction. As is well known $\left(F_{\text {exp }}\right)^{1 / 2}$, determines, for a constant wavelength, the angular width of an asymmetric reflection. ${ }^{10}$

We have chosen $\mathrm{Si}(333), \mathrm{Si}(444)$, and $\mathrm{Si}(555)$ reflections with $\phi=59^{\circ}$ for the first experiments and this gives the theoretical $F_{\text {exp }}$ versus $\theta_{B}-\phi$ shown in Fig. 2. This figure also shows the size of the illuminated footprint in the plane of reflection on the beam expander crystal for the case where $H=2 \mathrm{~mm}$. Expansion factors of $50-100$ can be

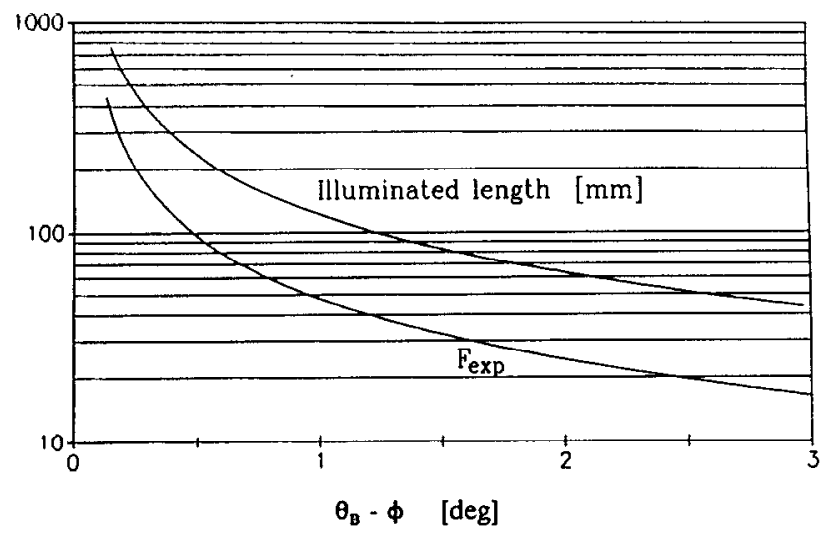

FIG. 2. $F_{\text {exp }}$ and illuminated length $L$ on beam expander crystal as a function of $\left(\theta_{B}-\phi\right), \phi$ is assumed to be $59^{\circ}$ and $H=2 \mathrm{~mm}$. achieved for $\theta_{B}-\phi$ in the range $0.5^{\circ}-1^{\circ}$ which are well above $\theta_{c}$, the critical angle for total reflection for the relevant wavelengths. Higher order correction to the classical theory of dynamical $\mathrm{x}$-ray diffraction is important for angles between the surface and the incident beam at or below $\theta_{c}{ }^{11}$

\section{BEAM EXPANDER CRYSTAL PREPARATION}

The crystals were sawed after careful alignment of the 111 planes relative to a fixturc fitting into the diamond saw ${ }^{12}$ at the Laboratory of Applied Physics at the Technical University of Denmark. The raw crystal was obtained from Topsil Semiconductor, Inc. It was a cylindrical Si boule $\sim 7.5 \mathrm{~cm}$ in diameter and $\sim 10 \mathrm{~cm}$ long with the 111 planes perpendicular to the axis of the cylinder. After sawing the crystals were lapped in a standard facility at Topsil to achieve a sufficiently flat surface. Finally, one of the faces of each crystal was polished. The dimension of crystal $A$ are $84 \times 38 \times 3.65 \mathrm{~mm}^{3}$ and those of crystal $B$ are $98 \times 38$ $\times 3.65 \mathrm{~mm}^{3}$. larger crystals from a $12.5 \mathrm{~cm}$ diameter and $30 \mathrm{~cm}$ long crystal boule are now being prepared.

\section{EXPERIMENTAL RESULTS}

The purpose of the first experiment was to establish the beam expander facility and test the performance of the two crystals $A$ and $B$. The beam expander unit consists of the monochromator axis and the bcam expander axis. The monochromator axis has a step resolution of 0.2 arcsec. The beam expander axis is supported on a rotatable arm (step resolution $1 \mathrm{mdeg}$ ) extending from the monochromator axis. The distance between the two axes along this arm can be varied. This construction allows the expanded beam to remain undeviated when different crystal reflections are used in the double reflection monochromatorbeam expander system. As a result, the optics under test need not be moved (except for alignment) when crystals with different $\phi$ values (see Fig. 1) or different reflections are used. A precise crystal translation (step resolution 10 $\mu \mathrm{m})$ perpendicular to its surface accurately positions the expander crystal in the beam from the monochromator.

Precise $( \pm 5 \mathrm{eV})$ energy calibration was accomplished by detecting the $K$-absorption edge from transmission measurements of thin foils of $\mathrm{Cu}, \mathrm{Zn}$, and $\mathrm{Ni}$. Following the selection of an appropriate energy with the premonochromator, the reflected beam from the monochromator was readily obtained. The beam expander crystal is carefully positioned to be parallel to the heam and to intercept half of the intensity reflected from the monochromator. Finally, the expanded beam is found by rotating the beam expander from the zero angle until the reflection is detected. This provides an accurate determination of $\theta_{B}-\phi$ (see Fig. 1). Since $\theta_{B}$ is known from the energy calibration this measurement determines the value of $\phi$. These are listed in Table I together with the values of $F_{\text {exp }}$ calculated from $\theta_{B}$ and $\phi$ for the energies and reflections used in the experiment.

The width of the slit (see Fig. 1) in the vertical direction was fixed to be $\sim 1 \mathrm{~mm}$ in all cases. This means that 
TABLE I. Overview of experimental data.

\begin{tabular}{|c|c|c|c|c|c|c|}
\hline \multirow[b]{2}{*}{$\begin{array}{l}\text { Crystal/ } \\
\text { reflection }\end{array}$} & \multirow[b]{2}{*}{$\begin{array}{c}E[\mathrm{keV}] / \\
\theta_{B}(\mathrm{deg})\end{array}$} & \multirow[b]{2}{*}{$\begin{array}{c}\text { Measured } \\
\phi(\mathrm{deg})\end{array}$} & \multirow[b]{2}{*}{$F_{\text {exp }}$} & \multirow{2}{*}{$\begin{array}{l}\text { Measured } \\
\text { width of } \\
\text { reflection } \\
\text { (arcsec) }\end{array}$} & \multicolumn{2}{|c|}{$\begin{array}{c}\text { Calculated width } \\
\text { of reflection (arcsec) }\end{array}$} \\
\hline & & & & & $\begin{array}{l}\text { polarization } \\
\text { out-of-plane }\end{array}$ & $\begin{array}{c}\text { polarization } \\
\text { in-plane }\end{array}$ \\
\hline$A / \mathrm{Si}(444)$ & $9.00 / 61.47$ & 59.04 & 20.3 & 10 & 12.5 & 6.8 \\
\hline$B / \mathrm{Si}(444)$ & $9.00 / 61.47$ & 59.06 & 20.5 & & & \\
\hline$B / \mathrm{Si}(444)$ & $9.14 / 59.93$ & 59.06 & 57.6 & & & \\
\hline$B / \mathrm{Si}(555)$ & $11.41 / 60.01$ & 59.06 & 52.7 & 4 & 6.5 & 3.4 \\
\hline
\end{tabular}

$\Delta \lambda / \lambda$, the wavelength resolution following the premonochromator, is given by $\Delta E / E=\Delta \lambda / \lambda$ $=\Delta \theta_{V} \cot \left[\theta_{B}(\mathbf{P M})\right]$ where $\theta_{B}(\mathbf{P M})$ is the Bragg angle in the $\mathrm{Si}(111)$ premonochromator and $\Delta \theta_{V}=2.86 \times 10^{-5}$ $\mathrm{rad}$ is the angular collimation of the synchrotron beam in the vertical direction. For $9 \mathrm{keV}$ this yields $\Delta \lambda / \lambda$ $\sim 1.3 \times 10^{-4}$. For $\mathrm{Si}(444)$ reflection at $9 \mathrm{keV}$ a $\Delta \lambda / \lambda$ of $9 \times 10^{-5}$ was measured, when a symmetrically cut crystal with a $\mathrm{Si}(444)$ reflection was used, in place of the asymmetrically cut beam expander crystal, together with the monochromator in the dispersive $(4,+4)$ setting. Since the Bragg angle for the reflections listed in Table $I$ is $\sim 60^{\circ}$, this implies that the horizontal collimation $\Delta \theta_{H} \sim 9$ $\times 10^{-5} \tan \left(60^{\circ}\right) \sim 33$ arcsec. The collimation must be the same in the expanded beam since the monochromator and beam expander crystal form a nondispersive system. Reducing the slit width in the vertical direction from $1 \mathrm{~mm}$ to $\sim 0.35 \mathrm{~mm}$ (the vertical source size) in future experiments, will reduce the $\Delta \lambda / \lambda$ obtained from the premonochromator to $\sim 4 \times 10^{-5}$ and, therefore, reduce the obtainable horizontal, angular collimation to $\sim 15$ arcsec. $F_{\text {exp }}$ was measured as a function of the horizontal slit width $H$ for crystal $B$ used in the 444 reflection at $9 \mathrm{keV}$. For the data shown in Fig. 3, values of $F_{\exp }$ are obtained by dividing the width of the observed expanded beam by $H$. The theoretical value of $F_{\exp }$ is 20.5 . The data show that near theoretical expansion is obtained for $H$ values up to $2 \mathrm{~mm}$ but reduced expansion is observed for $H=4 \mathrm{~mm}$. This is in good agreement with a horizontal source size of 2.8 mm. ${ }^{9}$

The measured rocking curve widths obtained by rotating the beam expander crystal through its reflection range may be directly related to predictions of dynamical diffraction theory. Measured rocking curve widths (FWHM) are given in Table I. For these measurements $H$ was adjusted to illuminate the entire length of the beam expander crystal. The angular widths of reflection in the asymmetric beam expander crystal which are calculated from dynamical diffraction theory, are also given in Table I for both in-plane and out-of-plane polarizations. The data in Table I show, as expected, that the measured reflection widths lie between these two values.

The intensity across the expanded beam measured in the one-dimensional PSD is shown in Fig. 4. In this case, the energy of the expanded beam was $6.85 \mathrm{keV}$ and the $\mathrm{Si}(333)$ reflection was used with a $H$ of $2 \mathrm{~mm}$ and a theoretical $F_{\text {exp }}$ of 53.3 (see Table I). This should give $L \simeq 121$ $\mathrm{mm}$. Since the crystal is only $98 \mathrm{~mm}$ long an expanded beam of only $98 \sin \left(\theta_{B}+\phi\right) \sim 86 \mathrm{~mm}$ (see Fig. 1) can be observed. Figure 4 shows that a reasonably constant intensity is observed along the detector for a length which corresponds to this value. The data in Figure 4 show that the width of the expanded beam is now limited only by the crystal size. We plan to produce crystals of length $>200$
Expansion Factor

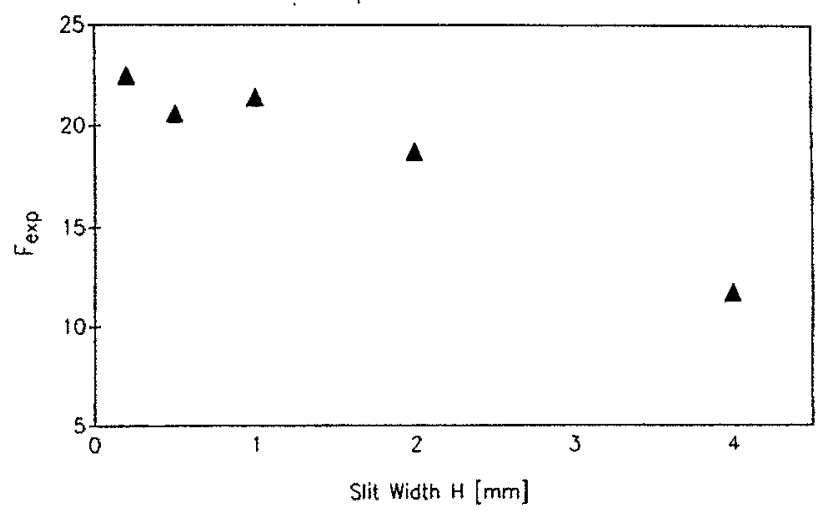

FIG. 3. Measured values $F_{\exp }$ vs slit width $H$ for a Si(444) reflection at 9 $\mathrm{keV}$.

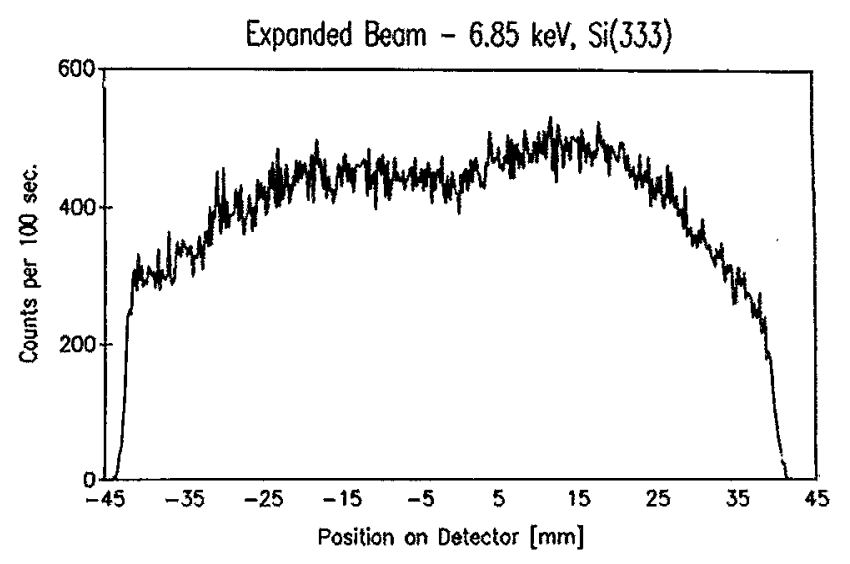

FIG. 4. The expanded beam reflected from $\mathrm{Si}(333)$ at $6.85 \mathrm{keV}$ detected in one-dimensional PSD. 
TABLE II. Energy table (keV) in $x$-ray telescope test.

\begin{tabular}{|c|c|c|c|c|c|}
\hline & $\phi[\mathrm{deg}]$ & 40 & 50 & 59 & 71 \\
\hline \multicolumn{6}{|c|}{ Reflection } \\
\hline $\mathrm{Si}(333)$ & & 9.13 & 7.69 & 6.88 & 6.25 \\
\hline $\mathrm{Si}(444)$ & & 12.17 & 10.25 & 9.18 & 8.34 \\
\hline $\mathrm{Si}(555)$ & & 15.22 & 12.81 & 11.47 & 10.42 \\
\hline \multicolumn{6}{|c|}{ Energies are calculated assuming $\theta_{B}-\phi=0.5^{\circ}$. } \\
\hline
\end{tabular}

$\mathrm{mm}$, and they will be used to obtain an expansion factor of $>100$ for $\left(\theta_{B}-\phi\right) \sim 0.5^{\circ}$. Finally, we measured a countrate in the expanded beam of $\sim 30 \mathrm{kHz}$ under the following conditions: Ring current $\sim 200 \mathrm{~mA} /$ multibunch mode, slit dimensions $2 \times 1 \mathrm{~mm}^{2}, E=9.14 \mathrm{keV}$, and a Si(444) reflection.

Generating the expanded beam using the arrangement in Fig. 1 is, of course, possible without the premonochromator. In that case, all the orders of reflection will be expanded simultaneously. In addition, the intensity in each order will be increased when compared with the arrangement which includes the premonochromator. This may be impractical for testing of $\mathrm{x}$-ray telescopes with a two-dimensional PSD developed especially for the low countrates encountered in $\mathrm{x}$-ray astronomy, Using the facility without the premonochromator will be very useful for the testing of large PSDs developed for special high countrate synchrotron beam lines. ${ }^{1}$

\section{X-RAY TELESCOPE CALIBRATION}

The detailed calibration of the SRG telescopes being produced at the Danish Space Research Institute ${ }^{2}$ will be performed using the beam expander facility at important energies between $\sim 5$ and $15 \mathrm{keV},{ }^{2}$ using the four beam expander crystals listed in Table II. The SRG telescopes (Table III) are described in detail in Ref. 2. The complete arrangement which will be used for the calibration of $x$-ray telescopes is shown in Fig. 5. A distance of $400 \mathrm{~mm}$ will be fixed between the axis of the beam entering hutch 8.4 and the center of the expanded beam. Not shown in Fig. 5 are the evacuated beam pipes which will be used between the various elements especially in the 8-m-long stretch from the telescope to the detector. The calibration data will be obtained by recording the image of the focused beam as the telescope is rotated around its optical axis. This produces the equivalent of a full aperature test of the telescope. A

TABLE III. Parameters of SRG telescope.

\begin{tabular}{lc}
\hline \hline Focal length & $8000 \mathrm{~mm}$ \\
Number of mirrors & $8 \times 154$ \\
Outer mirror diameter & $600 \mathrm{~mm}$ \\
Inner mirror diameter & $160 \mathrm{~mm}$ \\
Mirror length & $200 \mathrm{~mm}$ \\
Mirror thickness & $0.3 \mathrm{~mm}$ \\
Minimum mirror separation & $0.5 \mathrm{~mm}$ \\
Substrate material & $\mathrm{Al}$ \\
Refiecting material & $\mathrm{Au}$ \\
Mass of one telescope & $98 \mathrm{~kg}$ \\
\hline \hline
\end{tabular}

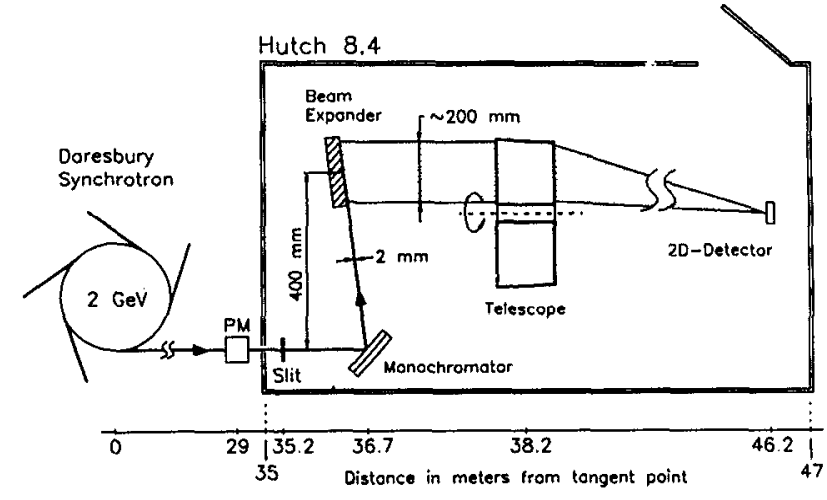

FIG. 5. Complete arrangement for the full aperture calibration of a high throughput $\mathrm{X}$-ray telescope.

precise rotation mechanism which supports the telescope has been constructed at the Danish Space Research Institute (DSRI) and initial tests indicate that the rotation of this mechanism contributes $<3$ arcsec to the image blur. ${ }^{13}$ This is much less than the requirement for any of the high throughput telescopes mentioned above.

Future high energy $x$-ray telescopes such as a proposed graded $d$-spacing multilayer telescope ${ }^{14}$ which has a substantial throughput up to $\sim 60 \mathrm{keV}$, can be evaluated in the beam expander facility since the synchrotron source will provide sufficient intensity at these energies.

\section{ACKNOWLEDGMENTS}

We thank $O$. Pedersen for the accurate alignment and cutting of the crystals. Futher we thank Topsil Semiconductor Inc., for lapping the crystals and $O$. Rupsch for his expertise in polishing the crystals.

\footnotetext{
${ }^{1}$ R. Lewis, N. Fore, C. Hall, B. Parker, I. Sumner, and J. Worgan, these proceedings; C. B.-Jørgensen, these proceedings.

${ }^{2}$ N. J. Westergaard, B. P. Byrnak, F. E. Christensen, P. Grundsøe, A. Hornstrup, S. Henrichsen, U. Henriksen, E. Jespersen, H. U. NørgaardNielsen, J. Polny, H. W. Schnopper, and P. Фrup, Opt. Eng. 29, 658 (1990).

${ }^{3}$ P. J. Serlemitsos, SPIE 830, 179 (1987).

${ }^{4}$ A. Wells et al. SPIE 1546, (1991).

${ }^{5}$ B. Aschenbach and H. Bräuninger, SPIE 982, 10 (1988)

${ }^{6}$ F. E. Christensen, B. P. Byrnak, A. Hornstrup, Zhu Shou-hua, and H. W. Schnopper, SPIE 1344, 14 (1990).

${ }^{7}$ E. H. Silver, A. Simionivici, S. E. Labov, R. Novick, P. Kaaret, C. Martin, T. Hamilton, M. C. Weisskopf, R. F. Elsner, J. Beeman, G. A. Chanan, G. Manzo, E. Costa, G. C. Perola, and G. W. Fraser SPIE 1160, 587 (1989).

${ }^{8}$ H. Wolter, Ann. Phys. NY 10, 94 (1952). H. Wolter, Ann. Phys. NY 10, 286 (1952).

${ }^{9} \mathrm{P}$. Quinn (private communication).

${ }^{10}$ W. H. Zachariassen, $X$-ray Diffraction in Crystals, (Dover, New York, 1935).

${ }^{11} \mathrm{O}$. Brümmer, H. R. Höche, and J. Nieber, Z. Naturforsch. 37a, 519 (1982).

${ }^{12}$ G. Christiansen, L. Gerward, and I. Alstrup, Acta. Crystallogr. A 31, 142 (1975).

${ }^{13}$ C. Nilsson, P. Frederiksen, Masters thesis, Danish Space Research Institute, 1991.

${ }^{14}$ F. E. Christensen, A. Hornstrup, N. J. Westergaard, H. W. Schnopper,
} J. Wood, and K. Parker, SPIE 1546, (1991). 